\title{
Amplitude noise and coherence degradation of femtosecond supercontinuum generation in all-normal-dispersion fibers
}

\author{
Etienne Genier, ${ }^{1,}{ }^{*}$ Patrick Bowen, ${ }^{1}$ Thibaut Sylvestre, ${ }^{2}$ \\ John Dudley, ${ }^{2}$ Peter Moselund ${ }^{1}$ and Ole Bang ${ }^{1,3}$ \\ ${ }^{1}$ NKT Photonics A/S, Blokken 84, DK-3460, Birkerød, Denmark \\ ${ }^{2}$ Institut FEMTO-ST, UMR 6174 CNRS-Université de Franche-Comté, 25030 Besançon, France \\ ${ }^{3}$ DTU Fotonik, Department of Photonics Engineering, Technical University of Denmark, $2800 \mathrm{Kgs}$. \\ Lyngby, Denmark \\ *etge@nktphotonics.com
}

\begin{abstract}
Supercontinuum (SC) generation via femtosecond pumping in all-normal dispersion (ANDi) fiber is predicted to offer completely coherent broadening mechanisms, potentially allowing for substantially reduced noise levels in comparison to those obtained when operating in the anomalous dispersion regime. However, previous studies that cover this topic typically treat only the one-photon-per-mode noise, not considering other technical noise contributions that will affect the stability of the pump laser and thus the coherence of the SC generation process. In this work, we discuss the various sources of noise that can be added to a numerical simulation, and provide numerical analysis showing that the coherence limits of ANDi SC generation are considerably reduced when amplitude and pulse duration noise are taken into account.
\end{abstract}

(C) 2018 Optical Society of America under the terms of the OSA Open Access Publishing Agreement

\section{Introduction}

Highly nonlinear photonic crystal fibers (PCFs) having all-normal-dispersion (ANDi) have only recently emerged as an attractive method to generate low-noise, octave-spanning supercontinua (SC) [1-4]. This is due to the fact that these PCFs are difficult to fabricate (e.g, requiring submicron holes), and that broadband low-noise ANDi SC generation requires high peak power femtosecond lasers. It has been shown that low-noise ANDi SC generation cannot be achieved using long pulses because the Raman effect is just as noisy as modulation instability [5]. Despite these obstacles, femtosecond ANDi SC generation has received significant attention because of its ability to generate pulses that can theoretically maintain their temporal coherence completely; a feature that is unachievable in the anomalous dispersion regime. This gives such systems potential in a range of fields including optical coherence tomography (OCT), optical metrology and spectroscopy [6-9].

The reason for this high coherence comes from the coherent spectral broadening mechanisms of self-phase modulation (SPM) and optical wave-breaking (OWB) [10,11]. In femtosecond pumped ANDi SC systems, these mechanisms are considerably more efficient than the incoherent nonlinear effects of noise-seeded modulation instability and stimulated Raman scattering [1], allowing for octave spanning SC generation in which these incoherent effects are completely suppressed for sufficiently short fiber lengths and input pulse durations [10,12].

Several theoretical studies about SC coherence have been reported in anomalous dispersion fiber, covering pulse durations from femtosecond up to continuous wave, and including many different kinds of noise such as one-photon-per-mode noise, induced by the quantum uncertainty of the energy of a single photon, Raman noise, and polarization noise [13-21]. This is not the case for numerical studies of ANDi SC generation, which have mostly considered only the one-photon-per-mode noise $[1,10,18]$, except for a very recent work of Gonzalo et al. [12], in 
which relative intensity noise (RIN) was experimentally and numerically compared, for ANDi SC generation with 170 fs pump pulses. In this study, it was found that one-photon-per-mode noise was insufficient to numerically describe the system, whereas adding $1 \%$ amplitude fluctuations of the laser gave good agreement with the experimental results. Significantly, this important fact was just mentioned for one specific pulse duration and fiber length and no specific simulation including the amplitude noise was shown and no general study conducted [12]. This behavior, in which the contribution of one-photon-per-mode noise diminishes and the influence of amplitude fluctuations increases in high-peak-power systems $(100 \mathrm{~kW})$ should not be surprising. However, while these technical noise sources have traditionally contributed little to the SC noise levels in incoherently broadened anomalous dispersion fiber SC [18, 22], coherently broadened ANDi SC require a new and comprehensive understanding of the noise sources emanating from the pump laser, for which mode-locked lasers and amplifiers are primarily responsible.

In this work, we aim to accurately describe the influence of the noise sources of a typical femtosecond mode-locked laser on SC generation, and with this, numerically investigate the coherence and RIN of the SC spectrum generated in an ANDi fiber pumped with fs pulses at 1054 $\mathrm{nm}$. We then demonstrate that the addition of this noise can degrade the coherence considerably, beyond that of the one-photon-per-mode noise and discuss the changes in spectral and average coherence with regards to the SC generation dynamics. These results show that the limits for high coherence, in terms of pulse duration and fiber length, suggested in previous publications, change substantially when technical noise is included. Indeed, we find that while a high coherence can be maintained for a pulse duration below $1.2 \mathrm{ps}$ in a system without technical laser noise and only with one polarization [10], a pulse duration of $\sim 50 \mathrm{fs}$ will begin to lose coherence when a relatively low noise pump laser is properly described. We re-iterate that we focus on single polarization SC generation because it allows us to more clearly demonstrate the impact of technical laser noise.

\section{Technical noise of a mode-locked fiber laser}

In this study, a one-dimensional generalized nonlinear Schrödinger equation is used to simulate pulse propagation of the envelope function $A=A(z, T)$, with initial condition $A(0, t)=$ $\sqrt{P_{0}} \operatorname{sech}\left(t / T_{0}\right)$, in a highly nonlinear single mode optical fiber. This includes dispersion (described by a Taylor expansion up to $\beta_{10}$ ), spectrally dependent linear loss $\alpha(\omega)$ and the nonlinear response of the material as well as the dispersion of the nonlinearity and the Raman response, $R(T)[18]$ :

$$
\frac{\partial A}{\partial z}=-\frac{\alpha(\omega)}{2} A+\sum_{k \geq 2} \frac{i^{k+1}}{k !} \beta_{k} \frac{\partial^{k} A}{\partial T^{k}}+i \gamma\left(1+i \tau_{0} \frac{\partial}{\partial T}\right)\left(A \int_{-\infty}^{+\infty} R\left(T^{\prime}\right)\left|A\left(z, T-T^{\prime}\right)\right|^{2} d T^{\prime}\right),
$$

where $\gamma$ is the nonlinear coefficient and $\tau_{0}=1 / \omega_{0}$ represents the characteristic timescale of self-steepening [18]. The Raman response is modelled using the real Raman gain profil as described in [23].

In this study, three sources of noise are described. The first, is the well-known quantum noise, which is modelled semi-classically through the addition of a noise seed at the input of one-photon-per-mode (OPPM) with a random phase on each spectral discretization bin [18]. This noise, in the frequency domain, is given by $\delta_{\mathrm{QN}}=\sqrt{\mathrm{hc} \mathrm{dT} \Omega_{m} / \lambda} \exp \left(2 i \pi \Phi\left[\Omega_{m}\right]\right)$, where $h$ is the Planck constant, $d t$ is the temporal bin and $\Phi\left(\Omega_{m}\right)$ is a random value corresponding to white noise uniformly distributed in the interval $[0,1]$ in each frequency bin $\Omega_{m}$. The second noise source is the laser amplitude noise described by : $\delta_{\mathrm{AN}}=\Psi$, where $\Psi$ represents a single random value for each input pulse extracted from a Gaussian distribution having an unit mean and a standard deviation equal to the rms amplitude noise of the laser, given by the laser manufacturer 
of the modelled laser. In this paper, we will consider a range of rms amplitude noise going from $0.1 \%$ to $2 \%$ ( $0.2 \%$ represents the rms value of an Onefive Origami 10 femtosecond laser and $1 \%$ a Fianium femtosecond laser both from NKT Photonics).

To fully consider the effects of amplitude noise, we should also consider what effect such changes in amplitude will have on the pulse duration, as this will subtly affect the efficiency of self-phase modulation, and thus the output spectral shape and width. To model this in our system, we notice a correlation between average power and spectral width of a Origami 10. Assuming the pulse remains approximately transform-limited, a correspondence between the change in bandwidth and pulse duration can be established from the time bandwidth product equation as shown in the following one:

$$
\delta T_{0}=\frac{0.315 \lambda_{0}^{2}}{c \Delta \lambda}
$$

where $\delta T_{0}$ represents the standard deviation extracted from a Gaussian distribution with a mean equal to the input pulse duration and $\Delta \lambda$ represents the new bandwidth induced by the amplitude noise. Finally, a linearized relation linking the change in average power and pulse duration is implemented on the scale of the 0.1-2 \% amplitude fluctuations, which in our case leads to the following relationship: $\delta T_{0}=-0.8 *\left(\delta_{A N}-1\right)$ fs. We note that this value is specific to the laser considered but can be generalized for other kinds of lasers. The third type of noise that we know to be present in our system is the phase noise induced timing jitter. However, as the physical mechanism for this noise is known to be induced by acoustic vibrations causing low frequency changes in cavity length we can see that this noise source will induce only a temporal offset from pulse to pulse [24]. As this will have no effect on the spectral coherence or RIN of the SC process, we will not include this noise source. Finally, our initial condition becomes :

$$
A(0, t)=\sqrt{P_{0}} \delta_{\mathrm{AN}} \operatorname{sech}\left(t /\left(T_{0}\left(1-0.8\left(\delta_{\mathrm{AN}}-1\right)\right)\right)\right)+F^{-1}\left(\delta_{\mathrm{QN}}\right),
$$

where $F^{-1}$ represent the inverse Fourier transform.

\section{Results}

As explained above, we want to focus on just the effect of laser technical noise compared to the conventionally used one-photon-per-mode noise and therefore use a single-polarization scalar code. This is still highly accurate for polarization maintaining (PM) fibers, which is why we choose the specific PM ANDi PCF NL-1050-NE-PM from NKT Photonics. This PCF has a relative hole size of $d / L=0.45$, a small hole-to-hole pitch of $1.44 \mu \mathrm{m}$, and a stress-induced birefringence of $4 \times 10^{-4}$. Its classical ANDi dispersion profile, shown in Fig 1.a), was calculated with COMSOL and confirmed experimentally in the region 900-1300 nm using white light interferometry. The measurements of dispersion were done without controlling polarization, which is typically sufficient for stress-induced birefringence that does not significantly alter the mode profile. As expected, the small holes of the PCF give a confinement loss edge significantly below the material loss edge, here found to be at $1450 \mathrm{~nm}$ using COMSOL (see Fig 1.a)). This will significantly influence the long wavelength part of the intensity and noise profiles, as we will see in the following. The dispersion has a minimum of $-13 \mathrm{ps} / \mathrm{nm} / \mathrm{km}$ at $1040 \mathrm{~nm}$ and is rather symmetrical within the low-loss window. However, we pump at $1054 \mathrm{~nm}$, because we want to consider realistic noise values of a specific laser - the Origami 10. 

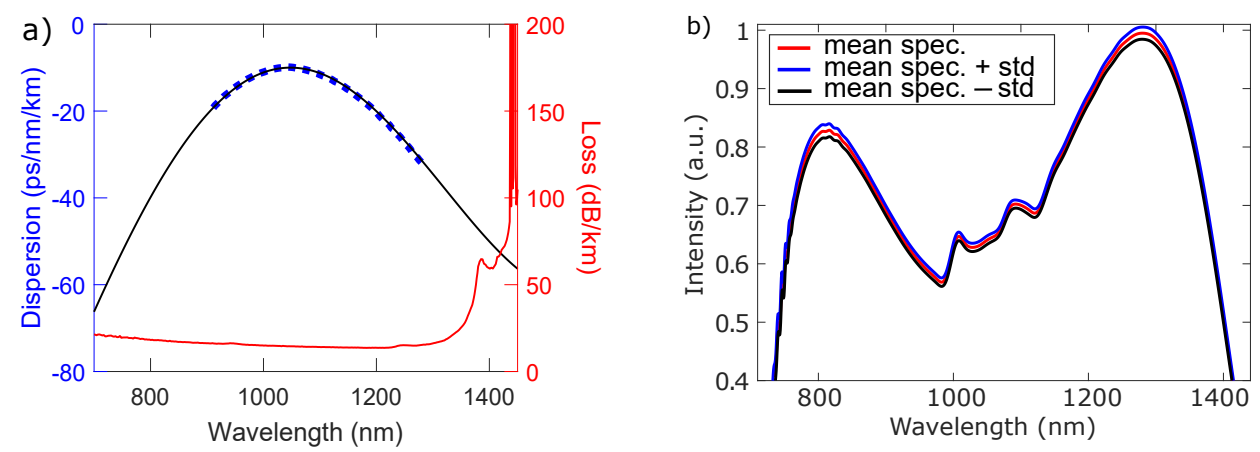

Fig 1.a) Measured (dashed blue) and modelled (solid black) dispersion curve, and fiber losses (solid red). Fig 1.b) Numerical supercontinuum spectra after $1 \mathrm{~m}$ of ANDi fiber showing fluctuation in spectral power and shape for $0.5 \%$ amplitude noise and a pulse duration standard deviation of $0.2 \mathrm{fs}$. The simulation uses an average pulse duration of $50 \mathrm{fs}$ and a peak power of $100 \mathrm{~kW}$ at $1054 \mathrm{~nm}$.

With an average pulse duration of $\tau=50 \mathrm{fs}$ and peak power $P_{\mathrm{P}}=100 \mathrm{~kW}$, the SC process is simulated over a length of $1 \mathrm{~m}$, and the effect of noise is shown in Fig 1.b). Here, we generated an ensemble of 20 simulated pulses then calculated and plotted for each wavelength of our ensemble the mean and the mean plus/minus the standard deviation value, we can see that for only a small amplitude noise contribution of $0.5 \%$ (pulse duration standard deviation of $0.2 \mathrm{fs}$ ), fluctuations in the SC spectrum are already noticeable on the edges.

In ANDi SC generation, it has been shown that the pulse duration, fiber length, and peak power have a critical influence on the noise properties $[10,12]$. To see the effect of amplitude noise with regards to these parameters, we cover a wide parameter space, simulating and calculating the spectrally averaged coherence. These are shown for a range of input pulse durations and fiber lengths, in Fig 2.a), where the amplitude noise is $0.3 \%$. As is typical of ANDi-SC systems, the coherence decreases when either pulse duration or fiber length is increased $[10,12]$. The coherence function and the spectrally averaged coherence are defined as $[10,18]$ :

$$
\left|g_{12}(\omega)\right|=\left|\frac{\left\langle\tilde{A}_{i}^{*}(\omega) \tilde{A}_{j}(\omega)\right\rangle_{i \neq j}}{\sqrt{\left\langle\left|\tilde{A}_{i}(\omega)\right|^{2}\right\rangle\left\langle\left|\tilde{A}_{j}(\omega)\right|^{2}\right\rangle}}\right|, \quad\left\langle\left|g_{12}\right|\right\rangle=\frac{\int_{0}^{\infty}\left|g_{12}(\omega)\right|\left\langle|\tilde{A}(\omega)|^{2}\right\rangle d \omega}{\int_{0}^{\infty}\left\langle|\tilde{A}(\omega)|^{2}\right\rangle d \omega} .
$$

However unlike typical studies, in Fig 2.a) we observe a considerably limited range of parameters where the noise is low. For $0.3 \%$ amplitude noise, a spectrally averaged coherence above 0.9 is only achieved for pulse durations below $100 \mathrm{fs}$. This is shown of the contour lines that crosses Fig 2.a), which corresponds to the pulse duration and lengths where $\left\langle g_{12}\right\rangle=0.9$. Figure 2.b) shows this again, as well as the contour lines at $\left\langle g_{12}\right\rangle=0.9$ for amplitude noise levels between $0.1-1 \%$ (pulse duration standard deviation going from $0.04 \mathrm{fs}$ to $0.4 \mathrm{fs}$ ), as shown on the graphic for amplitude noise higher than $1 \%$ an average coherence of 0.9 cannot be obtained, for any reasonable fiber length. From this, we can see clearly the dramatically limiting effect that the addition of standard laser noise levels to a numerical simulation have on the coherence parameter space. Significantly, Fig 2.b) does not show a contour line for the case when only one-photon-per-mode noise is present because there is no loss of coherence observable until soliton numbers around 600, corresponding in this case to a pulse duration of $1.2 \mathrm{ps}$, as shown by Heidt et al. [10]. 

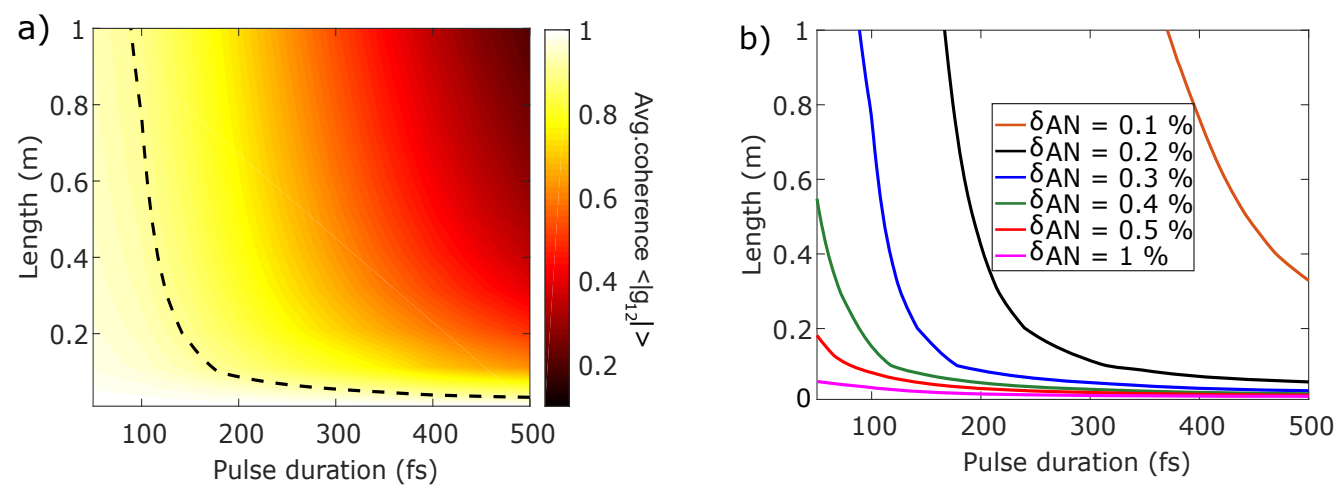

Fig 2.a) Average spectral coherence of SC pulse generated with $100 \mathrm{~kW}$ peak power pump pulses as a function of pump pulse duration and propagation distance including an amplitude noise value of $0.3 \%$ and a pulse duration standard deviation of $0.12 \mathrm{fs}$. The dotted line indicates where the spectrally averaged coherence is 0.9 . Fig $2 . b$ ) Evolution of the spectrally averaged coherence value of 0.9 for a range of amplitude noise value going from $0.1-1 \%$ and their pulse duration standard deviation associated.

Experimentally, it is typically the relative intensity noise (RIN), which is used to characterize the noise of an SC source. The frequency-dependent profile, $\operatorname{RIN}(\omega)$, is defined as [12]:

$$
R I N(\omega)=\sqrt{\left\langle\left(|\tilde{A}(\omega)|^{2}-\left\langle|\tilde{A}(\omega)|^{2}\right\rangle\right)^{2}\right\rangle} /\left\langle|\tilde{A}(\omega)|^{2}\right\rangle .
$$

Figure 3.a) shows this spectrally resolved $\operatorname{RIN}(\omega)$ for different levels of amplitude noise, and we see that in all cases a 50 fs pulse has low RIN (below $1 \%$ ) for the majority of the bandwidth. We can also define the average RIN as :

$$
\langle R I N\rangle=\int_{0}^{e d g e}|R I N(\omega)|\left\langle|\tilde{A}(\omega)|^{2}\right\rangle d \omega / \int_{0}^{e d g e}\left\langle|\tilde{A}(\omega)|^{2}\right\rangle d \omega,
$$

where edge is defined as the wavelength where $|A(\lambda=e d g e)|<-30 \mathrm{~dB}$. An $0.5 \%$ amplitude noise than gives $\langle R I N\rangle=1.7 \%$, which corresponds to an average coherence of 0.82 . This is in contrast to the much lower RIN of around $0.2 \%$ which we see for most of the wavelength span of Fig 3.a), thus indicating much higher RIN on the spectral edges, going to $24 \%$ on the short-wavelength edge and $1.4 \%$ on the long-wavelength edge. We note that this asymmetric behavior on the edges is due to the confinement loss edge at $1450 \mathrm{~nm}$, which removes the longer wavelengths. Indeed, if we omit fiber losses from our model, we find a RIN value on the long-wavelength edge of $23 \%$ which is in close agreement to the $24 \%$ obtained in the short-wavelength edge. We note that the impact of the long-wavelength loss edge was studied previously showing suppression of rogue waves in anomalous dispersion supercontinuum generation [25]. While this observation, like our own, corresponds to a reduction in RIN of the supercontinuum spectrum at long wavelengths, it would appear there is no correlation with our study as the rogue waves generated in that study come about through a collisional multi-solitons process which is not possible in an ANDi fiber. This strong spectral dependence on RIN provides context to the result to Fig 2.a) b), showing they cannot be interpreted in isolation because spectral edge is considerably higher than the majority of the spectrum (one order of magnitude). Again, the dynamics can be further visualized in Fig 3.b) showing the evolution of the RIN profile along the $1 \mathrm{~m}$ fiber. Finally, the ripple aspect of the RIN spectrum is due to the quantum noise, and indeed, if we omit this noise these ripples will be suppressed and a smoother spectrum 

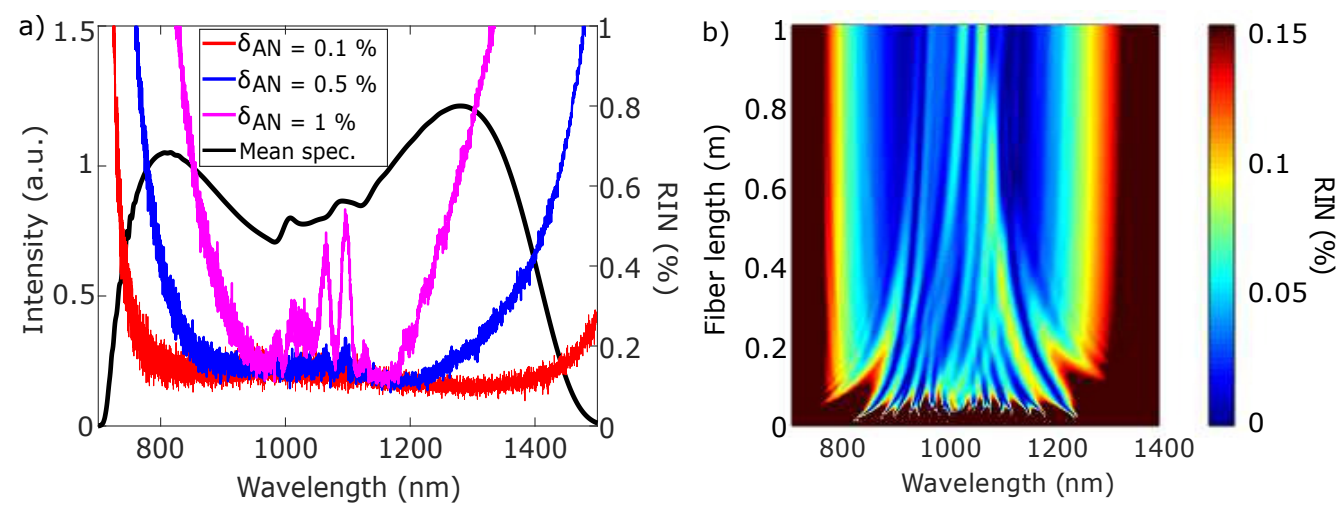

Fig 3.a) RIN profile for different amplitude noise values (color curves) and mean spectrum of an ensemble of 20 pulses (bold black curve) after $1 \mathrm{~m}$ of fiber with $100 \mathrm{~kW}$ peak power, $50 \mathrm{fs}$ pulse duration at $1054 \mathrm{~nm}$ for an amplitude noise value of $0.5 \%$ and a pulse duration standard deviation of 0.2 fs. Fig 3.b) Evolution of the RIN along the fiber length for an amplitude noise value of 0.5 $\%$ and a pulse duration standard deviation of $0.2 \mathrm{fs}$. NB: The color map has a dynamic range limited to a RIN equal to $0.15 \%$, meaning RIN data is only visible for wavelengths $780-1320 \mathrm{~nm}$.

appears. Furthermore, we note that while the average RIN increases during the propagation, the spectrally resolved RIN in the central region of the spectrum decreases with the propagation, with the higher RIN quotients being pushed to the edges of the spectrum.

Another noticeable feature is the relationship between the spectrum evolution and the RIN structure. Indeed, Fig 4.a) shows the RIN profile and the mean spectrum after a $10 \mathrm{~cm}$ fiber propagation. We can notice a correlation between the structure added by the SPM on the spectrum and the structure of RIN. These appear to have equal periodicity, suggesting they are related, however the exact position of each peak in the RIN structure does not seem to correspond to any particular point in the SPM structure. The two biggest peaks located respectively at $920 \mathrm{~nm}$ and $1180 \mathrm{~nm}$ correspond to the junction between the SPM and OWB generated parts of the spectrum. As shown on Fig 3.a), during the propagation the RIN spectrum becomes smoother (i.e. the SPM RIN peaks disappear) thanks to the OWB and the dispersion. Furthermore, for a one meter of propagation the spectrum is stable (like the RIN one) and only few peaks are still presents compare to Fig 3.a) which correspond to the junction between the SPM and OWB generated parts of the spectrum, specifically corresponding to the edges of the broadest SPM oscillation.

Figure 4.b) shows the RIN spectrum after $10 \mathrm{~cm}$ of propagation for several input noise types. The position of the fringes is slightly different as a function of the input noise because the change in the amplitude and/or the pulse duration will change the SPM efficiency. Also, We can clearly notice the RIN spectrum including both amplitude and pulse duration noise is much lower than considering either amplitude noise or pulse duration noise only. Furthermore, The RIN level of each situation is explained through the input noise value: $\delta_{A N}$ being higher than $\delta_{T_{0}}$, it makes sense that the red curve is higher than the pink one. About the blue curve, because there is an opposite relationship between amplitude and pulse duration noise the total noise $\left(\delta_{T_{0}}=-0.8 \delta_{A N}\right)$ amount will be lower than the sum of each one. It could be understood by ... 

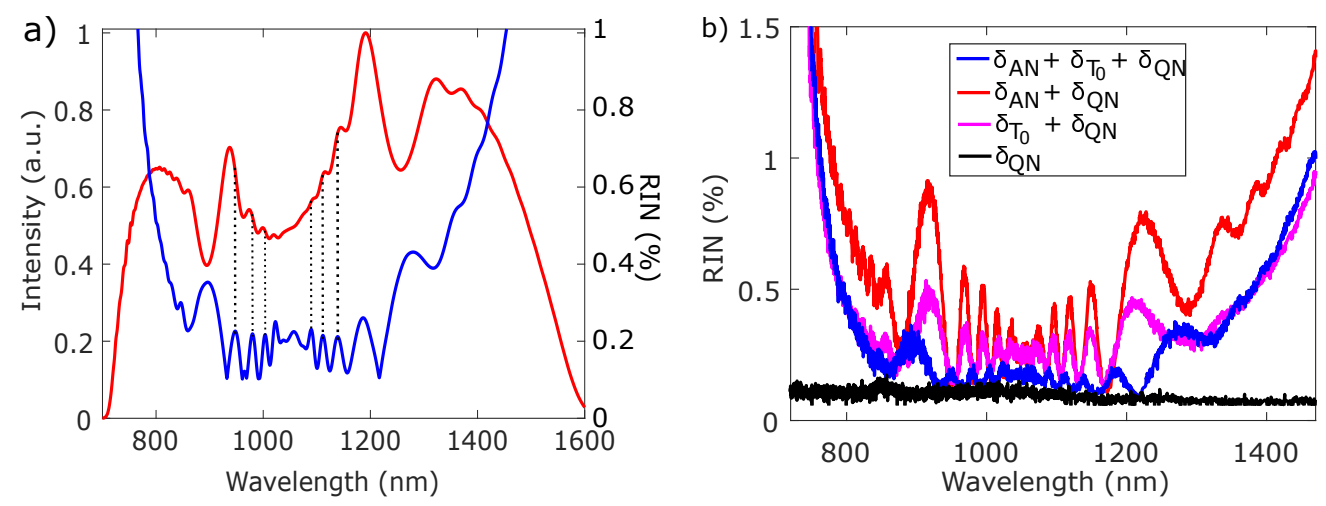

Fig 4.a) RIN profile (blue line) and mean spectrum (red line) of an ensemble of 20 pulses after $10 \mathrm{~cm}$ of fiber with $100 \mathrm{~kW}$ peak power, $50 \mathrm{fs}$ pulse duration at $1054 \mathrm{~nm}$ for an amplitude noise value of $0.5 \%$ and a pulse duration standard deviation of 0.2 fs. Fig 4.b) RIN spectrum as a function of the input noise : OPPM only (black line), amplitude noise plus OPPM noise (red line), pulse duration noise plus OPPM (pink line) and amplitude noise plus pulse duration noise and OPPM (blue line).

\section{Conclusion}

A detailed numerical study of the amplitude noise impact on the SC generation coherence in ANDi PCFs under high peak power femtosecond pumping at $1054 \mathrm{~nm}$ was presented, where it was shown that considering a nominal value of amplitude noise drastically affects the SC coherence. Indeed, when only the one-photon-per-mode noise model is used, the coherence starts to degrade with a pulse duration around $1.2 \mathrm{ps}$ while if we also consider an amplitude noise value of $0.5 \%$ on the pump laser, as may be found in a typical mode-locked laser, the degradation starts at a pulse duration of $\sim 50 \mathrm{fs}$. Specifically, it was shown that the SC RIN increases significantly on the spectral edges (higher than $24 \%$ ), converse to the middle part of the spectrum where it remained low (lower than $0.2 \%$ ). In this middle region, novel RIN dynamics were observed, with structures following the SPM and other features of the SC spectrum. Furthermore, we found that considering both pulse duration and amplitude noise led to a RIN level lower than each one considered separately. Such a study on finer details of SC noise in ANDi fiber is of substantial value to potential applications such as OCT and metrology, which require ultra-low-noise SC light sources. Indeed, this study constitutes the first in-depth look into the effect of technical noise sources on the ANDi SC process and provides grounds for further research to achieve a better understanding of these physically complex processes.

\section{Acknowledgment}

The research project leading to this work has received funding from the European Union's Horizon 2020 research and innovation programme under the Marie Sklodowska-Curie grant agreement No 722380, SUPUVIR, and grant agreement No 7326, GALAHAD.

\section{References}

1. A. M. Heidt, A. Hartung, G. W. Bosman, P. Krok, E. G. Rohwer, H. Schwoerer, and H. Bartelt, "Coherent octave spanning near-infrared and visible supercontinuum generation in all-normal dispersion photonic crystal fibers," Opt. Express 19, 3775-3787 (2011).

2. A. Hartung, A. Heidt, and H. Bartelt, "Design of all-normal dispersion microstructured optical fibers for pulsepreserving supercontinuum generation,” Opt. Express 19, 7742-7749 (2011). 
3. L. Liu, T. Cheng, K. Nagasaka, H. Tong, G. Qin, T. Suzuki, and Y. Ohishi, "Coherent mid-infrared supercontinuum generation in all-solid chalcogenide microstructured fibers with all-normal dispersion," Opt. Lett. 41, 392-395 (2016).

4. R. Alfano, "The Supercontinuum Laser Source," 3rd ed. (Springer, 2016).

5. U. Møller, O. Bang, "Intensity noise in normal-pumped picoseconds supercontinuum generation, where higher-order Raman lines cross into the anomalous dispersion regime," Electron. Lett. 49, 63-65 (2013).

6. M. Maria, I.B. Gonzalo, T. Feuchter, M. Denninger, P.M. Moselund, L. Leick, O. Bang, A. Podoleanu, "Qswitch-pumped supercontinuum for ultra-high resolution optical coherence tomography," Opt. Lett. 42, 4744-4747 (2017).

7. T. Udem, R. Holzwarth and T. Hänsch, "Optical frequency metrology," Nature 416, 233-237 (2002).

8. M.K Dasa, C. Markos, M. Maria, C.R. Petersen, P.M. Moselund, O. Bang, "High-pulse energy supercontinuum laser for high-resolution spectroscopic photoacoustic imaging of lipids in the 1650-1850 nm region," Biomed. Opt. Express 9, 1762-1770 (2018).

9. C.R. Petersen, N. Prtljaga, M. Farries, J. Ward, B. Napier, G.R. Lloyd, J. Nqllqlq, N. Stone, O. Bang, "Mid-infrared multispectral tissue imaging using a chalcogenide fiber supercontinuumsource," Opt. Lett. 43, 999-1002 (2018).

10. A. M. Heidt, J. S. Feehan, J. H. V. Price, and T Feurer, "Limits of coherent supercontinuum generation in normal dispersion fibers," J. Opt. Soc. Am. B 34, 764-775 (2017).

11. C. Finot, B. Kibler, L. Provost, and S. Wabnitz, "Beneficial impact of wave-breaking for coherent continuum formation in normally dispersive nonlinear fibers," J. Opt. Soc. Am. B 25, 1938-1948 (2008).

12. I. Gonzalo, R. Engelsholm, M. Sørensen, and O. Bang, "Polarization noise places severe constraints on coherence of all-normal dispersion femtosecond supercontinuum generation," Sci. Rep. 8, 6579 (2018).

13. F. Li, Q. Li, J. Yuan, and P. K. A. Wai, "Highly coherent supercontinuum generation with picosecond pulses by using self-similar compression," Opt. Express 22, 27339-27354 (2014).

14. M. H. Frosz, O. Bang, and A. Bjarklev, "Soliton collision and Raman gain regimes in continuous-wave pumped supercontinuum generation," Opt. Express 14, 9391-9407 (2006).

15. A. Mussot, E. Lantz, H. Maillotte, T. Sylvestre, C. Finot, and S. Pitois, "Spectral broadening of a partially coherent CW laser beam in single-mode optical fibers," Opt. Express 12, 2838-2843 (2004).

16. S. T. Sørensen, C. Larsen, U. Møller, P. M. Moselund, C. L. Thomsen, and O. Bang, "The role of phase coherence in seeded supercontinuum generation," Opt. Express 20, 22886-22894 (2012).

17. Z. Zhu and T. G. Brown, "Polarization properties of supercontinuum spectra generated in birefringent photonic crystal fibers," J. Opt. Soc. Am. B 21, 249-257 (2004).

18. J.M. Dudley, G. Genty, S. Coen, "Supercontinuum generation in photonic crystal fiber," Rev. Mod. Phys. 78, 1135-1184 (2006).

19. J. M. Dudley and S. Coen, "Coherence properties of supercontinuum spectra generated in photonic crystal and tapered optical fibers," Opt. Lett. 27, 1180-1182 (2002).

20. M. H. Frosz, "Validation of input-noise model for simulations of supercontinuum generation and rogue waves," Opt. Express 18, 14778-14787 (2010).

21. K. L. Corwin, N. R. Newbury, J.M. Dudley, S. Coen, S. A. Diddams, B. R. Washburn, K. Weber and R. S. Windeler, "Fundamental amplitude noise limitations to supercontinuum spectra generated in a microstructured fiber," Applied Physics B, 77, 269-277 (2003).

22. U. Møller, S. T. Sørensen, C. Jakobsen, J. Johansen, P. M. Moselund, C. L. Thomsen, and O. Bang, "Power dependence of supercontinuum noise in uniform and tapered PCFs," Opt. Express 20, 2851-2857 (2012).

23. I. Gonzalo and O. Bang, "Role of the Raman gain in the noise dynamics of all-normal dispersion silica fiber supercontinuum generation ," J. Opt. Soc. Am. B 35, 2102-2110 (2018).

24. O. Svelto, "Principles of lasers," 5th ed. (Springer, 2010).

25. D. Buccollero, H. Steffensen, H. Ebendorff-Heidepriem, T. monro, and O. Bang, "Midinfrared optical rogue waves in soft glass photonic crystal fiber," Opt. Express 19, 17973-17978 (2011). 